\title{
Extra Supplement: In the Beginning
}

Author(s): Charles Macpherson

Source: The Musical Times and Singing Class Circular, Vol. 42, No. 706 (Dec. 1, 1901), pp. 112

\section{Published by: Musical Times Publications Ltd.}

Stable URL: http://www.jstor.org/stable/3369256

Accessed: 27-06-2016 09:46 UTC

\footnotetext{
Your use of the JSTOR archive indicates your acceptance of the Terms \& Conditions of Use, available at http://about.jstor.org/terms
}

JSTOR is a not-for-profit service that helps scholars, researchers, and students discover, use, and build upon a wide range of content in a trusted digital archive. We use information technology and tools to increase productivity and facilitate new forms of scholarship. For more information about JSTOR, please contact support@jstor.org.

Musical Times Publications Ltd. is collaborating with JSTOR to digitize, preserve and extend access to The Musical Times and Singing Class Circular 


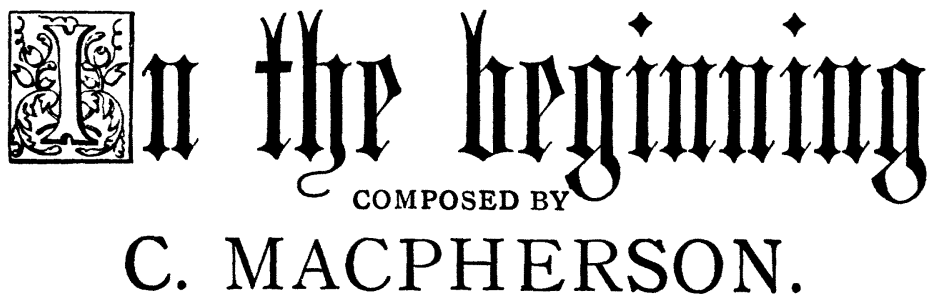

SIXTEEN VOLUMES NOW READY, BOUND IN CLOTH, PRICE 7s. EACH.

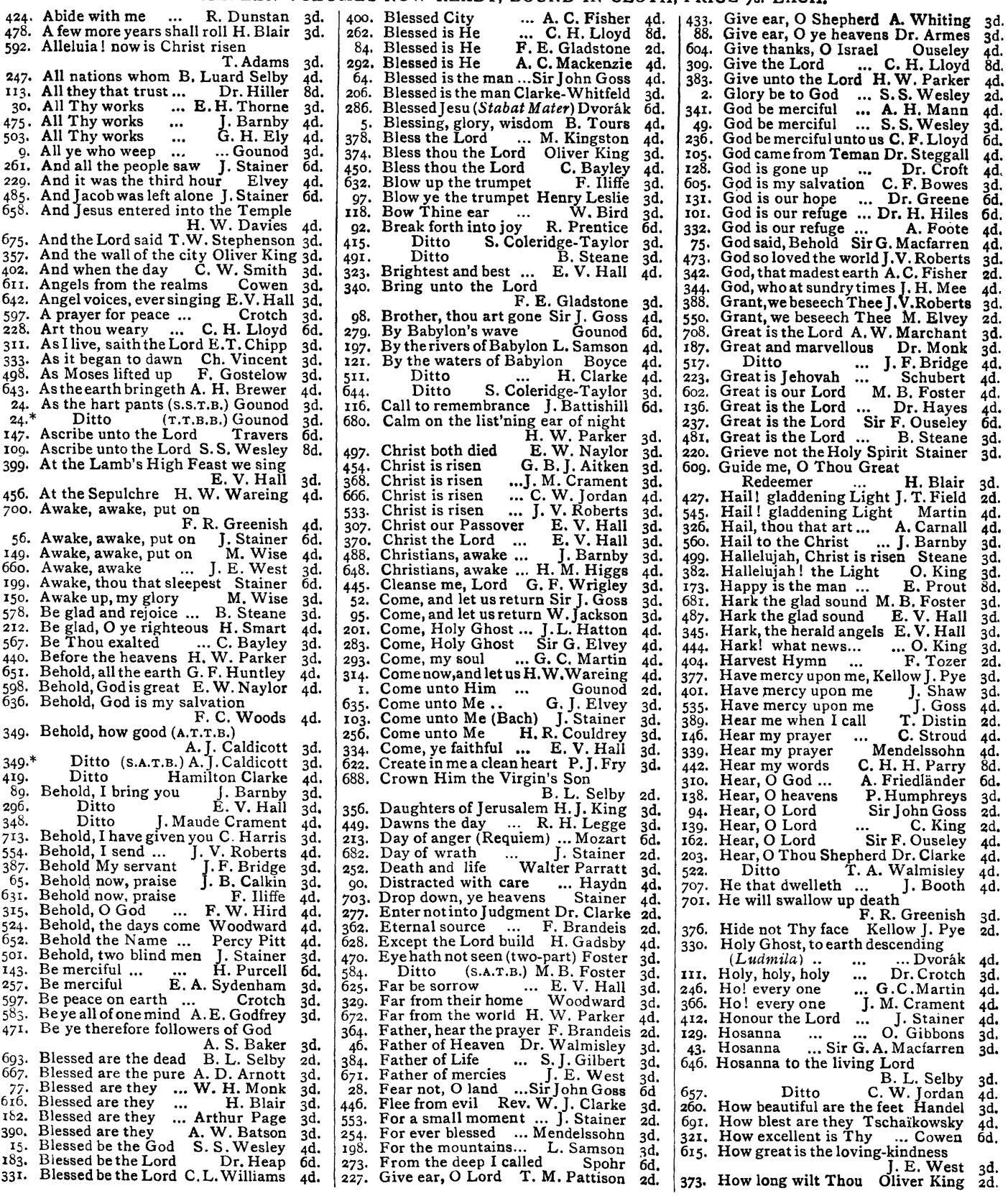

\section{LONDON: NOVELLO AND COMPANY, Limited AND \\ NOVELLO, EWER AND CO., NEW YORK.}

Copyright, 1901, by Novello and Company, Limited. 
The Musical Times,

EXTRA SUPPLEMENT.

December 1, 1901 .

\section{IN THE BEGINNING}

FULL ANTHEM FOR CHRISTMAS

Gen. i. 1, 27; ii. I5; Rom. v. I2; St. John iii. I6; and some Verses by the Rev. BERNARD REYNolds, M.A., Prebendary of St. Paul's Cathedral.

\section{CHARLES MACPHERSON}

Sub-Organist of St. Paul's Cathedral.
Price Fourpence.

London: NOVEllo AND COMPANY, Limited; and NOVELlo, EWER AND CO., New York.
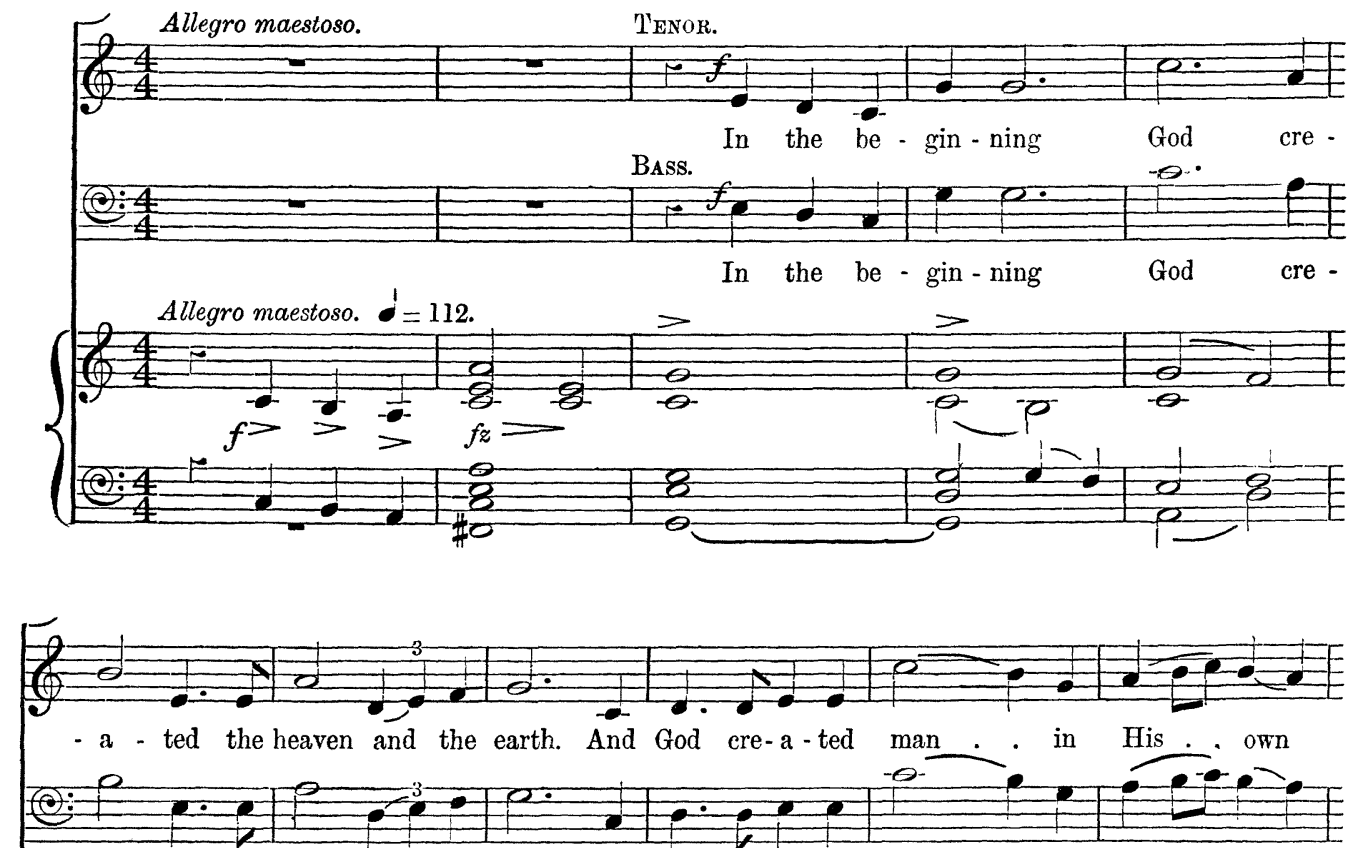

- a - ted the heaven and the earth. And God cre-a-ted man . . in His . . own
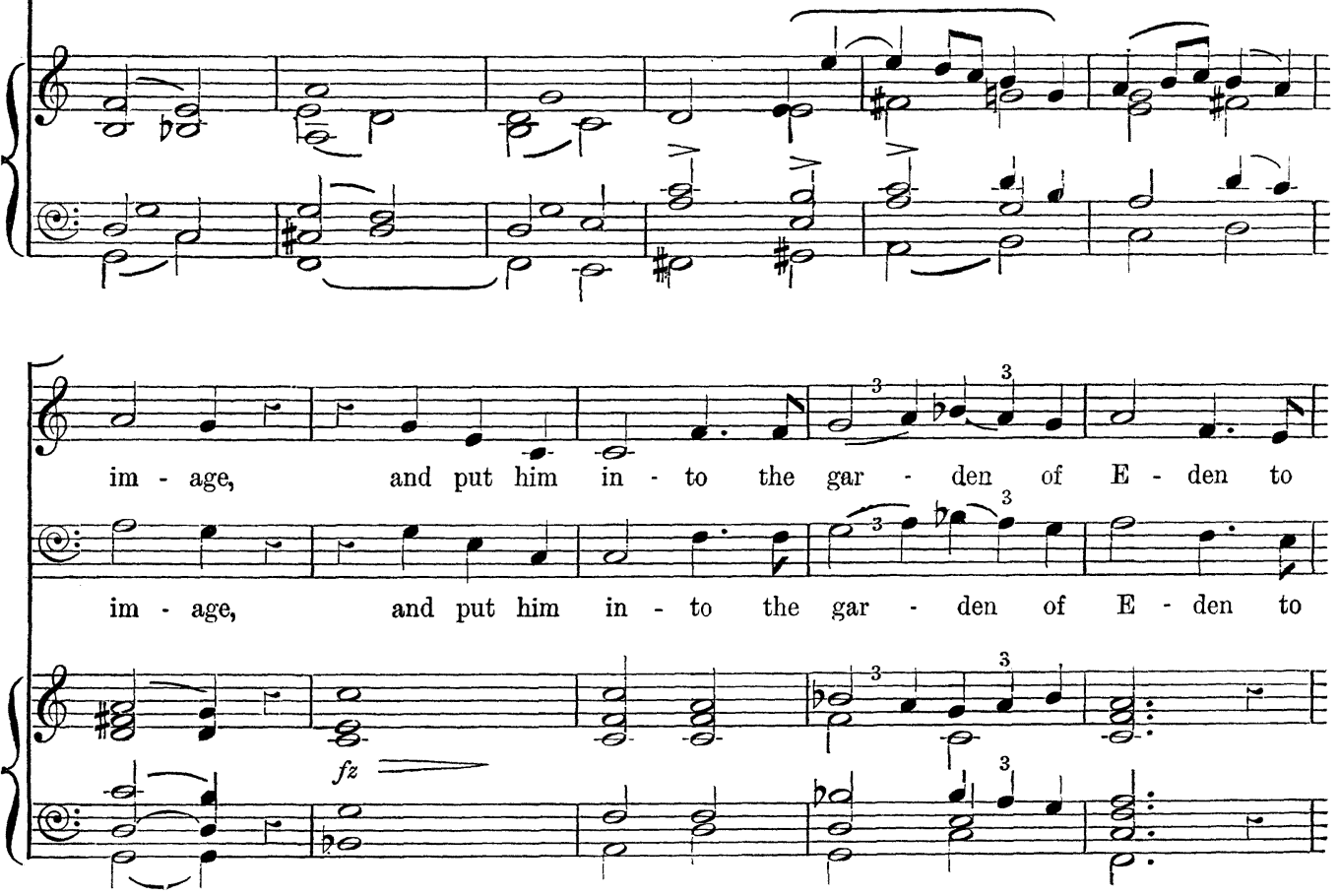

Copyright, 1901, by Novello and Company, Linited.

(2) 

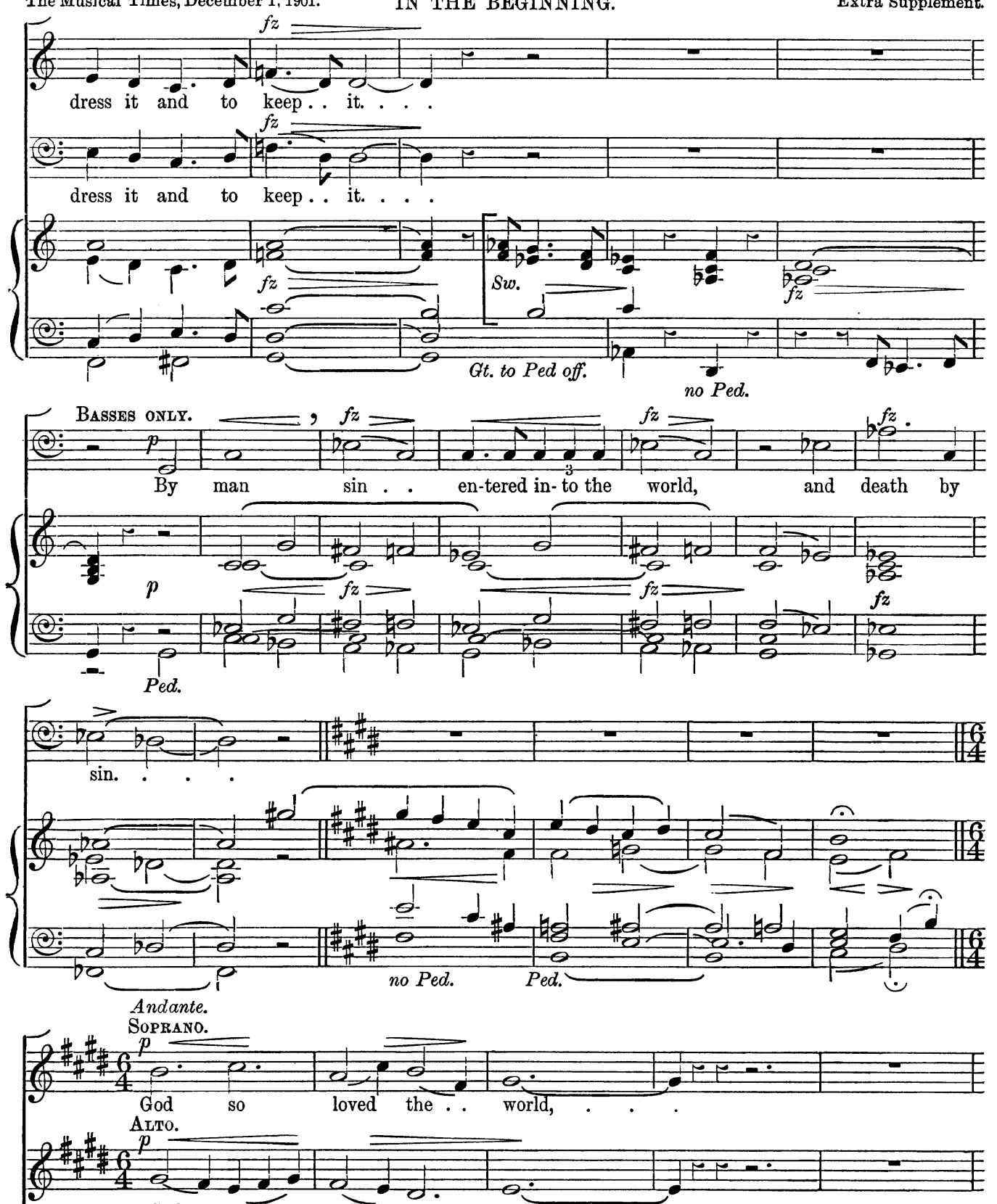

God .. so . loved the

Tenor.

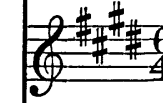

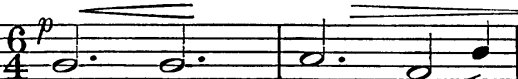

God loved

Bass.

(20)

BASS.

the . 

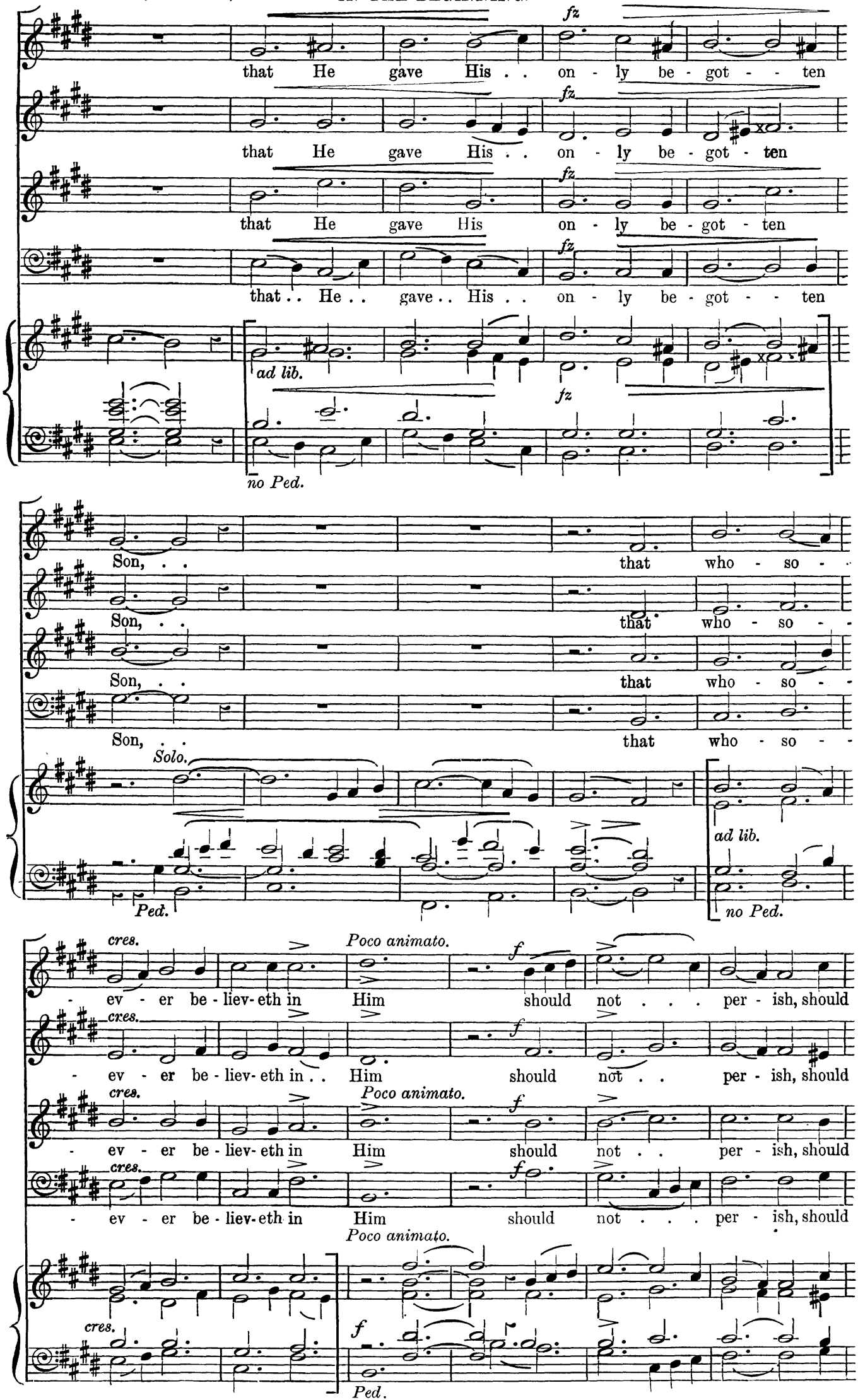

(4) 

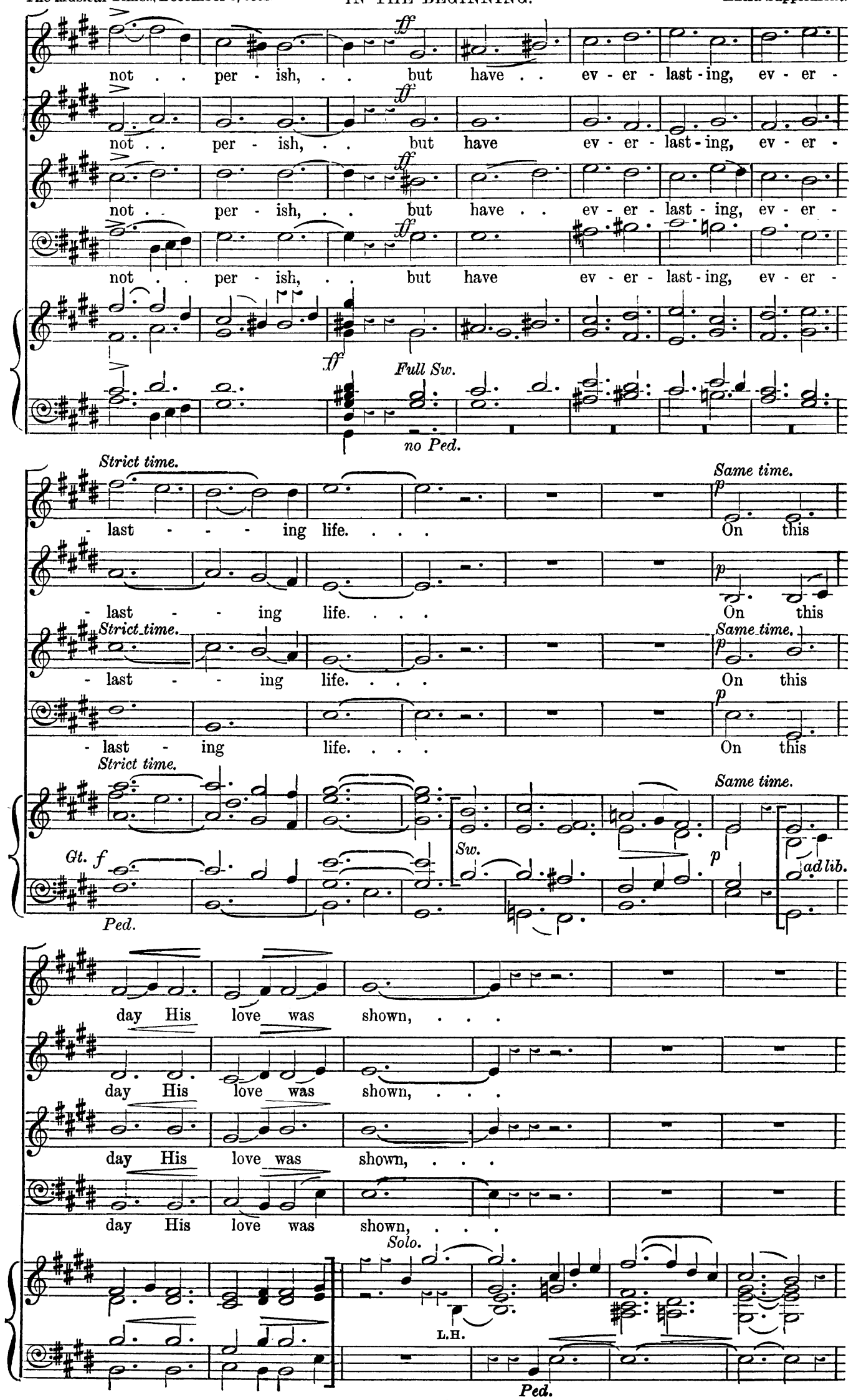

(5) 

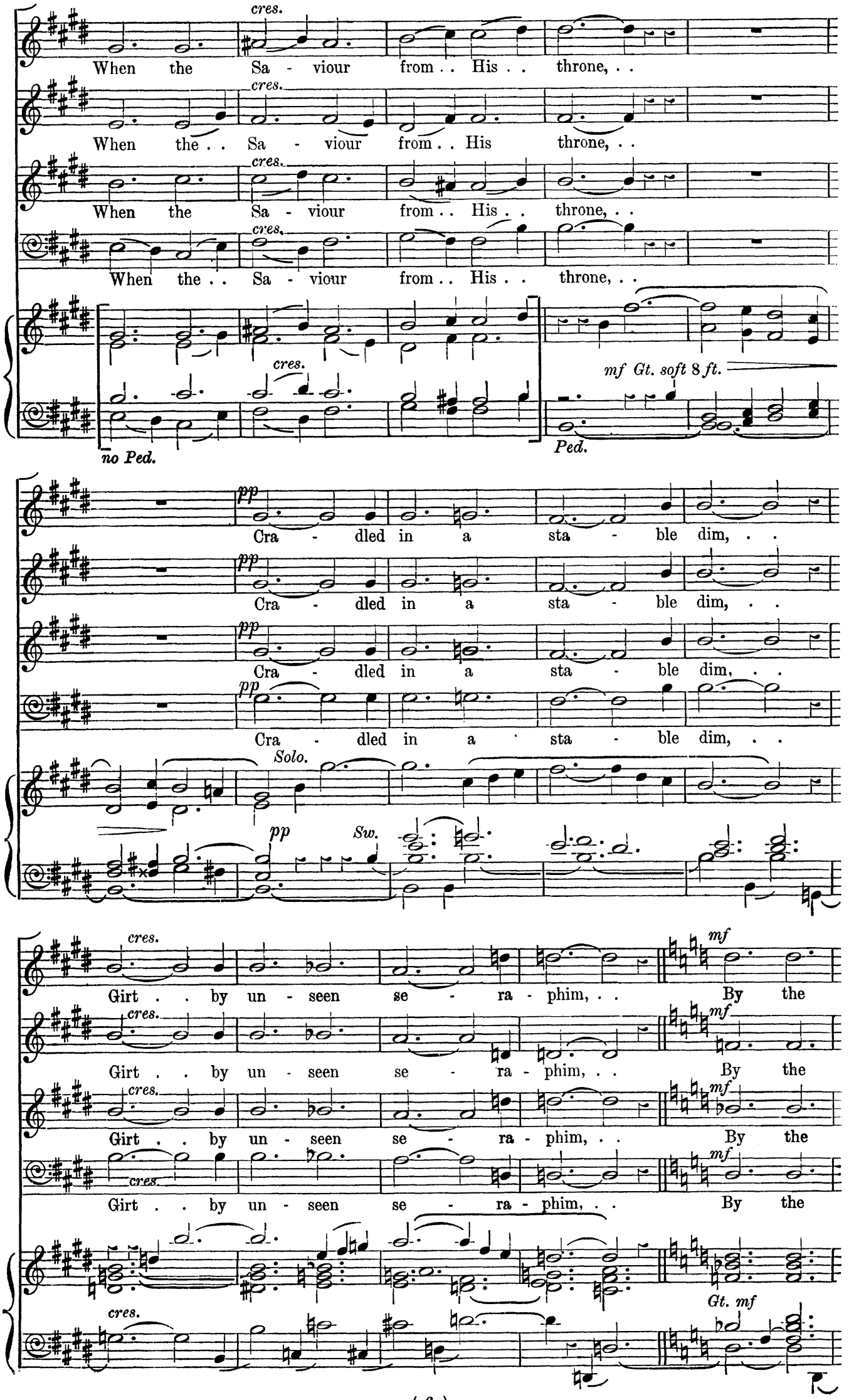

(6) 

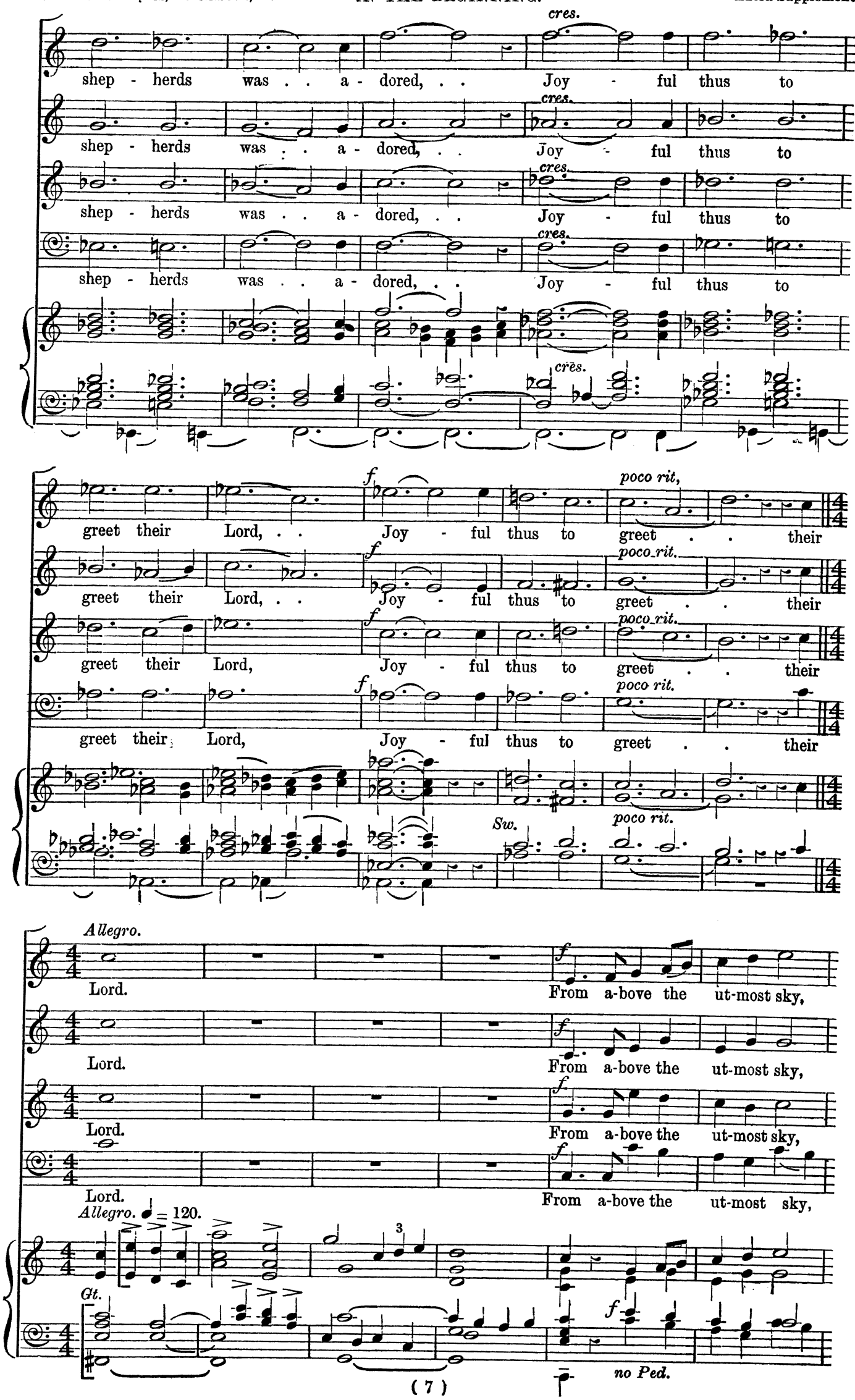

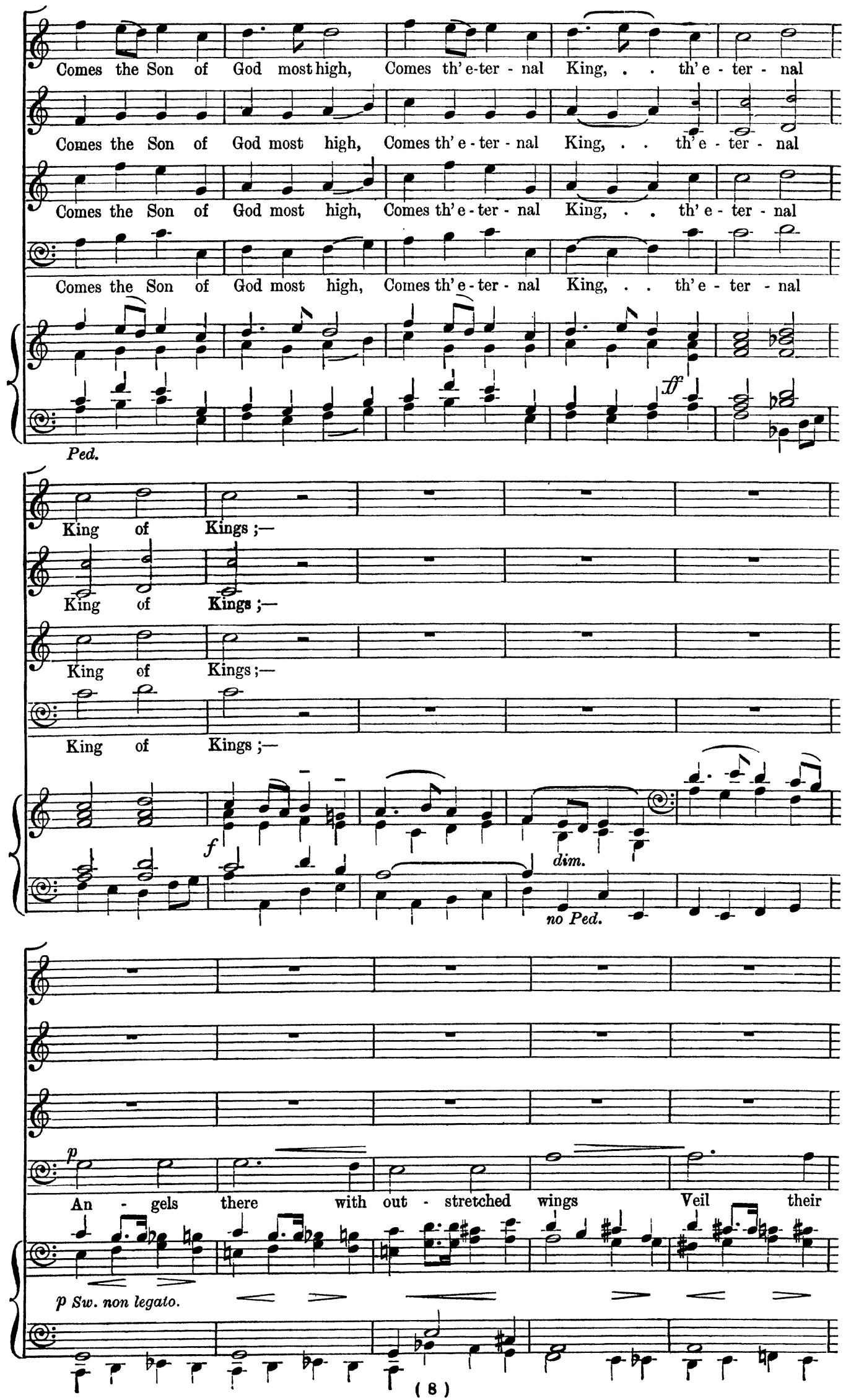

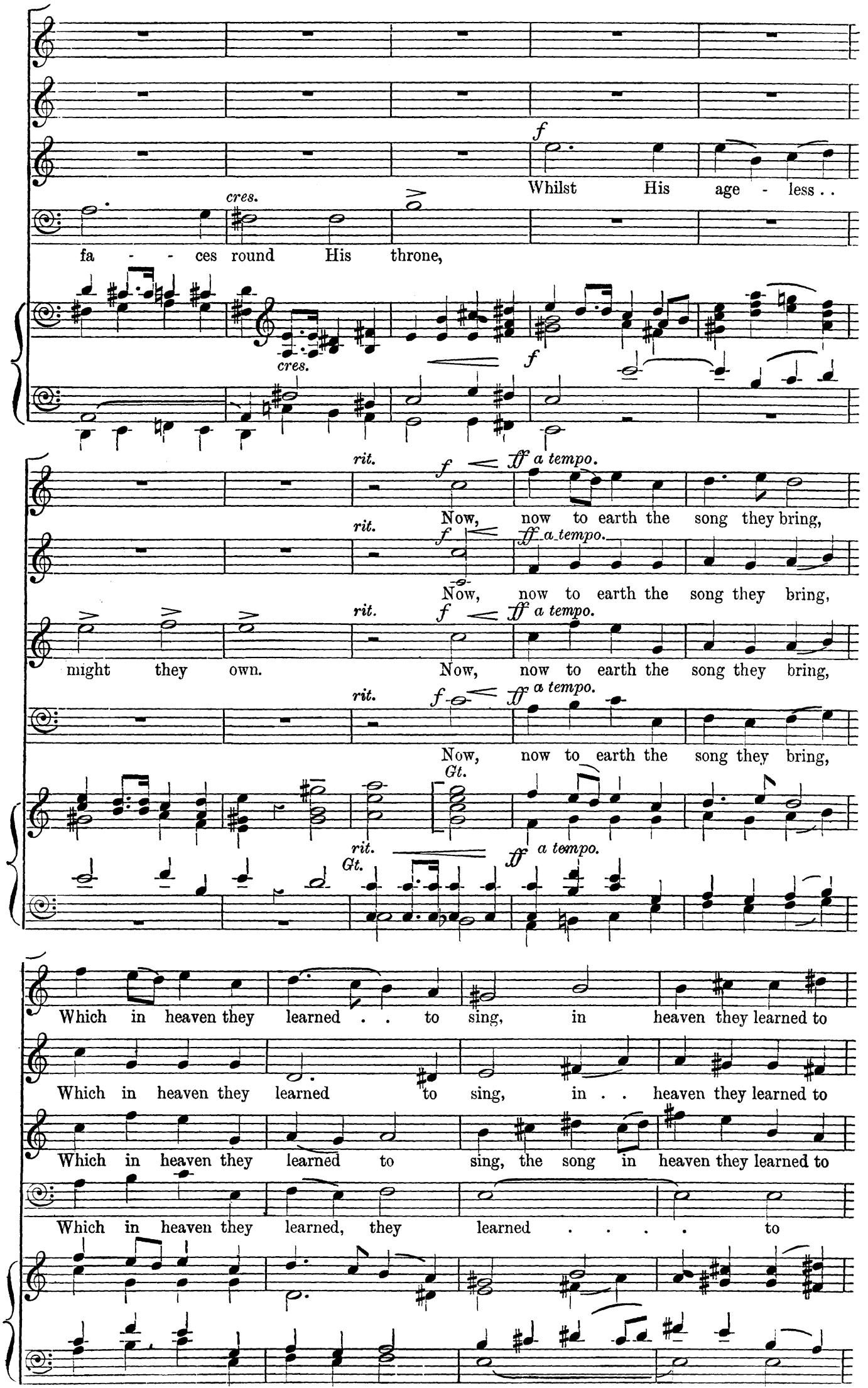

(9) 
The Musical Times. December 1, 1901.

IN THE BEGINNING.

Extra Supplement.
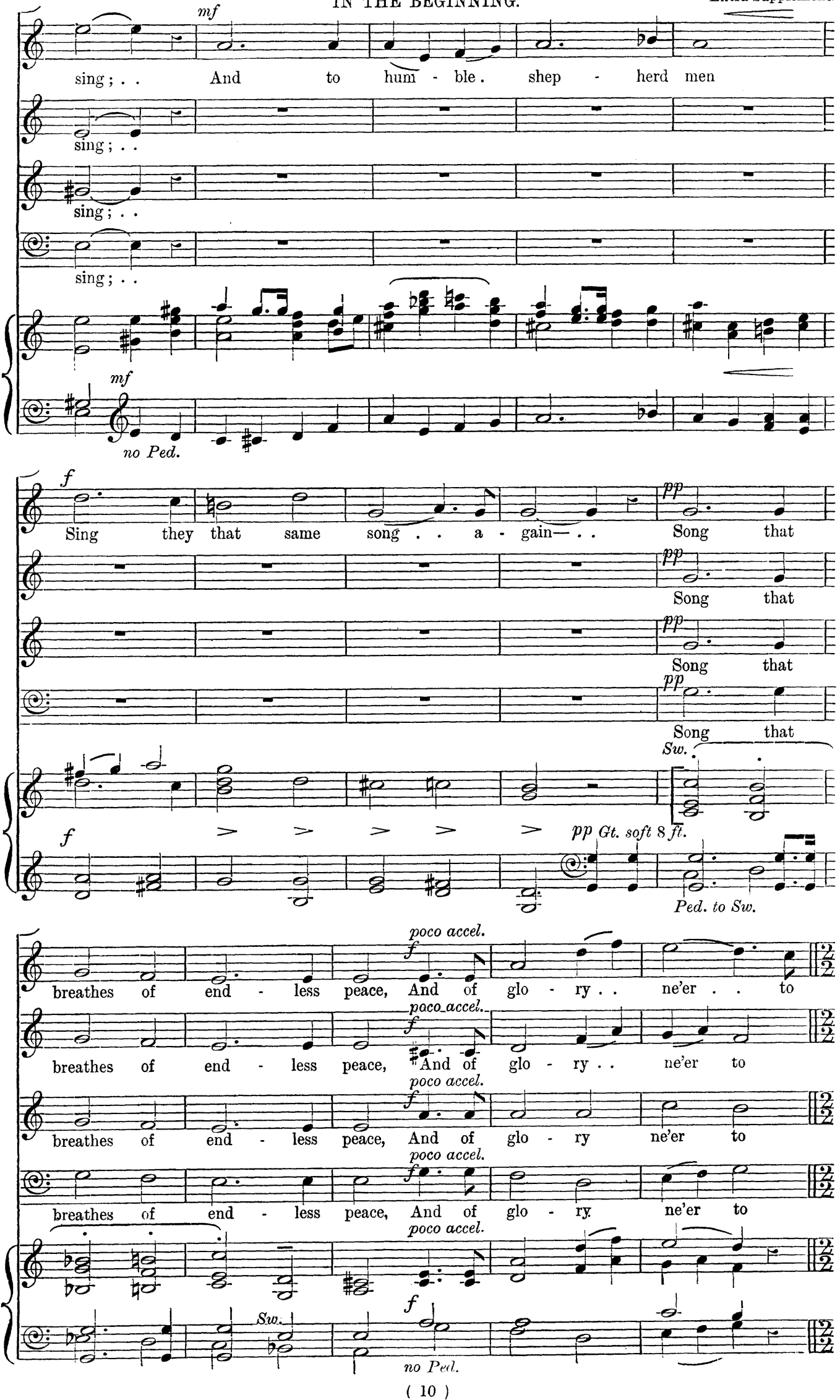

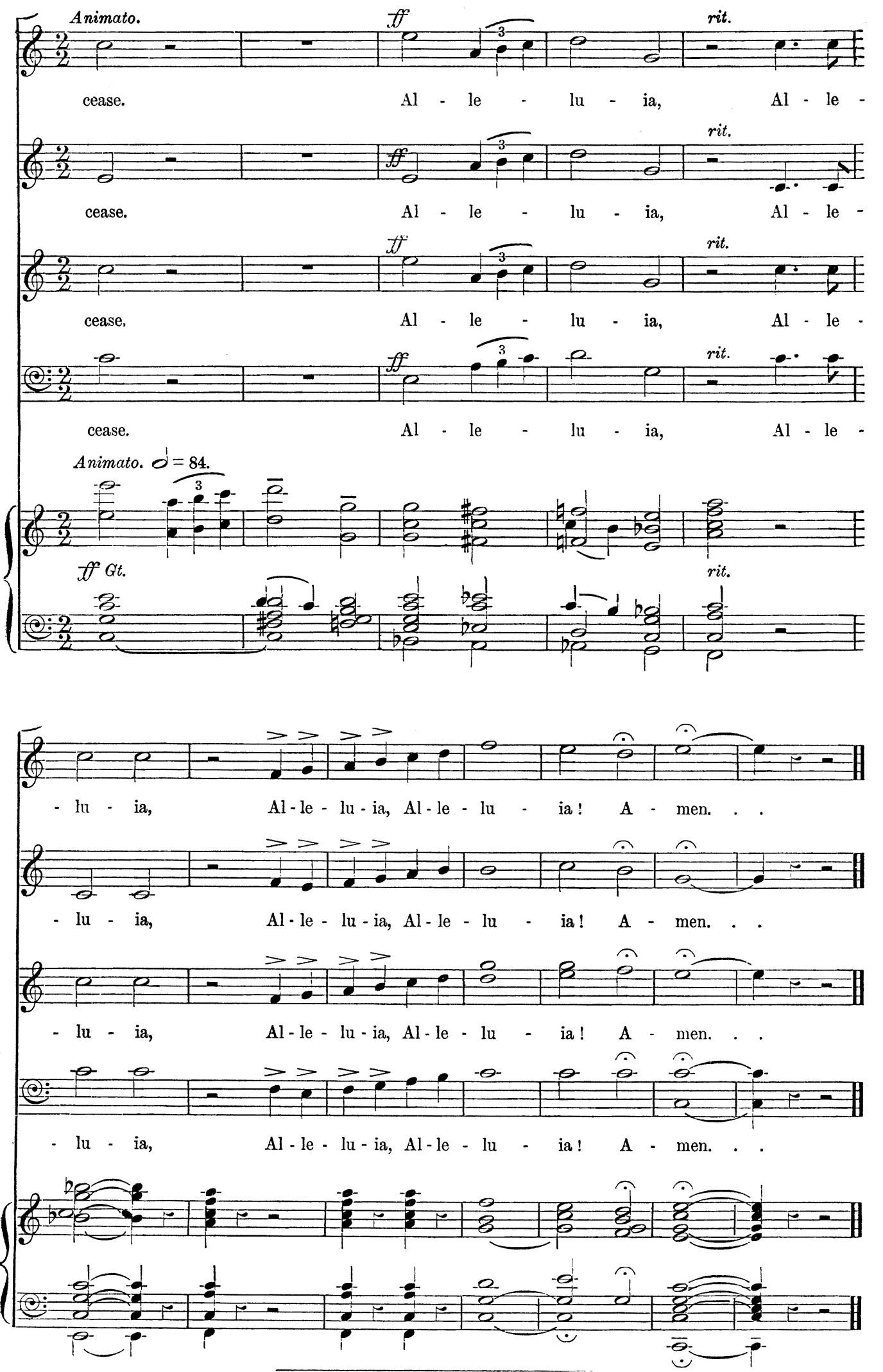

Also published in Novello's Tonic Sol-fa Series, No. 1232, price $1 \frac{1}{2} d$.

(11) 


\section{NOVELLO'S OCTAVO ANTHEMS-Continued.}

647. How lovely are 104. How lovely are 539. I am Alpha C. Salaman 3d. ... Spohr 8d. 623. I am He that liveth T. Adams 4d. 664. I am the Resurrection ... Croft $3 d$. C62. I am the Resurrection R. Rogers 4d. 608. I am well pleased J. Rheinberger 3d 120. I beheld, and lo ... Dr. Blow 6d. 280. I beheld, and lo ... ... Elvey $6 \mathrm{~d}$. 495. I came not to call C. Vincent 3 d. 207. I cried unto the Lord Dr. Heap 4 d. 537. I declare to you ... Cruickshank 4d. r68. I desired wisdom ... J. Stainer 6d. 230. I did call upon the Lord Pattison 4d. II7. I have set God ... Dr. Blake 6d. 130. I have set God ... J. Goldwin 3d. 420. I have set God Hamilton Clarke 4d. 122. I have surely built ... Dr. Boyce 4d. 219. I have surely built $T$. T. Trimnell $4 \mathrm{~d}$. 590. I heard a great voice G. F. Cobb 3d 396. I heard a voice Sir John Goss 2d. 171. I saw the Lord ... J. Stainer 6d. 114. I was glad ...

32. I was glad ...... E. Horsley $6 \mathrm{~d}$. 379. I was glad ... T. T. Trimnell 4d. r19. I was in the spirit ... Dr. Blow 6d. 205. I will alway give thanks Dr. Clarke $3^{\text {d }}$ 73. I will cry unto God Dr. Steggall 3d. 502. I will extol Thee C. M. Hudson 4 d. 29. I will give thanks ... J. Barnby 4 d. 156. I will give thanks ...E. J. Hopkins 6d. 568. I will give thanks ... Mozart 2d. 674. I will give you rain H.W.Wareing $4 \mathrm{~d}$ 225. I will go unto ...Dr.Gauntlett 2d. 59r. I will go unto the altar C. Harris $3 \mathrm{~d}$. 437. I will greatly rejoice Cruickshank 4 d. I95. I will lay me down... H. Gadsby 209. I will lay me down... Dr. H. Hiles 3 d. 195. I will lay me down A. C. Edwards 3d. 125. I will love Thee, O Lord J. Clark 4d. 194. I will love Thee, O Lord my strength ... Matthew Kingston 4d. 78. I will magnify Thee J. B. Calkin 4 d. 27. I will magnify Thee Sir John Goss 3 d. 153. I will magnify Thee ... J. Shaw 3 d. 405. I will magnify Thee ... O. King 4 d. 633. I will magnify Thee ... F. Iliffe 4 d. 55. I will mention ... Sir A. Sullivan 6d. 575. I will not leave you ... B. Steane 2d. 519. I will open rivers E. Pettman 3d. $37 \mathrm{I}$. I will set His dominion H.W.Parker $4 \mathrm{~d}$. I00. I will sing a new song Dr. Armes $8 \mathrm{~d}$ 608. I wil sing of the mercies J. Booth $3 \mathrm{~d}$. 134. I will sing of Thy power Greene 4d. 192. I will sing unto the Lord Wareing 3 d.

6. I will wash my hands E. J. Hopkins 3 d. 7ro. If any man hath not $H$. W. Davies $4 d$.

53. If we believe that Jesus died Goss 3 d. 453. If ye love $\mathrm{Me} H$. W. Wareing $3 \mathrm{~d}$. 544. If ye love $\mathrm{Me} \quad \ldots \quad \ldots$ B. Steane $2 d$. 469. If ye then be risen M, B. Foster $3 d$. 58. If ye then be risen ... Dr. Naylor $3 \mathrm{~d}$. 6r. In Christ dwelleth Sir John Goss 3 d. 6rg. In every place incense J.:E. West $3 \mathrm{~d}$. 655. In heavenly love ... H. Parker $3 \mathrm{~d}$. 403. In my Father's house Crament $3 \mathrm{~d}$. I02. In sweet consent ... E. H.Thorne 3 d. 278. In that day $\ldots \quad \ldots$ Sir G. Elvey $8 d$. 582. In the beginning $\ldots \quad$ F. Tozer $4 \mathrm{~d}$. 33. In Thee, O Lord ... B. Tours $3 d$. 148. In Thee, O Lord ... J. Weldon $3 \mathrm{~d}$. 385. In Thee, O Lord S. C. Taylor 3d. 338. In the fear of the Lord J.V. Roberts 3 d. 282. In the Lord ... Sir R. Stewart 6d. 659. In the Lord put I my trust

467. Is it nothing (2-pt.) M. B. Foster 3 d. 57I. Ditto (4 voices) M. B. Foster 3d. 91. It came even to pass Ouseley $4 \mathrm{~d}$. 180. It is a good thing ... J. Barnby 6d. 23I. It is a good thing T. M. Pattison 4 d. 215. It shall come to pass Dr. Garrett 6d. 397. Jesu, lover of my soul ...F. Iliffe Id. 455. Jesus Christ is risen Oliver King $4 \mathrm{~d}$.
654. Jesu, Thou joy ... $\quad$ E. H. Davies $3 \mathrm{~d}$. 548. Joy in harvest ... ... B. Steane $3 \mathrm{~d}$. 7. Judge me, O God ... Mendelssohn 4d. 677. Just Judge of Heaven ... Garrett 6d. 6r4. Justorum animæ $\quad \ldots \quad \ldots$ Byrd $3 \mathrm{~d}$. 581. Kings shall be thy nursing fathers

\section{G. C. Martin}

37. Lead, kindly Light... J. Stainer 4 d. 425. Lead, kindly Light... R. Dunstan 3d. 528. Lead, kindly Light C. L. Naylor 4d. 589. Lead, kindly Light D.Pughe-Evans $3 d$. 706. Let all the world C. W. Jordan 4d. 132. Let God arise ... Dr. Greene 6d. 375. Let God arise T. T. Trimnell 4d. 346. Let my complaint ...E. H. Thorne 3d. 509. Let not thine hand... J. Stainer 3d. 438. Let not your heart ... M. B. Foster 3d. 438.* Let not your heart M. B. Foster 3d. 226. Let the peace of God J.Stainer $4 \mathrm{~d}$. 565. Let the righteous ... R. F. Lloyd $3 \mathrm{~d}$. 328. Let the words of my A. D. Culley 3 d. 494. Let Thy merciful ears W. B. Bell 2 d. 308. Let us now praise ... E. H. Thorne 3d. 96. Lift up thine eyes ....Sir John Goss 6d. 18. Lift up your heads... J. L. Hopkins $1 \frac{1}{2}$ d. 409. Ditto S. Coleridge-Taylor 3d. 343. Lift up your hearts ... J. Barnby 4d. 408. Lighten our darkness G. R. Vicars 2d. 595. Light of the world ... E. Elgar 3d. 393. Like as the hart Thomas Adams 3d. 530. Lo, God, our God ... B. Haynes 3d. 7Ir. Look on the fields C. Macpherson 3 d. 639. Look upon the rainbow T. Adams $3 d$. 165. Lord, how are they... H. Clarke 6d. 391. Lord, I have loved... ....F. Iliffe 3 d. 54. Lord, let me know mine end Goss $3 \mathrm{~d}$. $35 \mathrm{r}$. Lord of all power ... J. Barnby 2 d. 459. Lord of our life $\ldots . \quad$ J. T. Field 3d. 566. Lord of life ... A. C. Mackenzie 404. Lord of the rich and golden F.Tozer $2 d$. 4II. Lord of the Harvest J. Barnby $4 \mathrm{~d}$. 3I8. Lord, Thou art God... J. Stainer 8d. 434. Lord, Thou hast ... A. Whiting 3d. 274. Lord, what love have I Dr. Steggall 6d. 267. Lord, who shall dwell Dr. Roberts 4 d. 335. Lo, summer comes again J.Stainer $6 \mathrm{~d}$. 504. Lo! the winter B. Farebrother 3d. 350. Magnify His Name... G. C. Martin $4^{\text {d. }}$ 290. Make a joyful noise A.C.Mackenzie $6 \mathrm{~d}$. 108. Make me a clean heart J. Barnby 3 d. 43r. Ditto A. W. Batson 3d. 436. Man goeth forth ... A. Carnall 3d. 694. Man that is born S. S. Wesley 2d. 222. Me ye have bereaved $c$. Morales $3 \mathrm{~d}$. 527. Mercy and truth are met J. Stainer $3 \mathrm{~d}$. 2II. Mine eyes look unto Thee H. Baker 3 d. 500. Miserere mei, Deus J. Barnby 3d. 665. Ditto ... Novello $2 \mathrm{~d}$. 46. Ditto J. Stainer 3d. 518. Ditto E. Pettman x 1 512. My beloved spake ... H. Purcell 6d. 428. My God, I love Thee G. J. Bennett 3d. 6I7. My God, I thank Thee E.H.Lemare 3d. 10. My God, my God ... Mendelssohn 6d. 288. My God, look upon...J. L. Hopkins $3 d$. 460. My heart was glad... A. Carnall $4 \mathrm{~d}$. 56. My heart is inditing M. B. Foster 199. My hope is in the 406. My mouth shall speak J. E. West 4 d. 190. My soul is weary ...Dr. Beckwith 4 d. 586. My soul truly waiteth B. Steane 2d. 295. My soul, wait thou still F. J. Read 4d. 629. Nearer, my God, to Thee T. Adams 3d. 210. Not unto us, O Lord H. Gadsby $6 \mathrm{~d}$. 558. Not unto us ... J. E. West 4 d. 592. Now is Christ risen T. Adams 3d. 612. Now is come salvation C. Harris $3^{d}$ 695. Now late on the Sabbath S. Coleridge-Taylor $3 d$ 353. My heart is fixed W. Cruickshank $4 \mathrm{~d}$

505. O all ye people ... H. Purcell 3d 506. O be joyful in the Lord G. Martin 6d. 217. O clap your hands ...T.T.Trimnell 3d 133. O clap your hands ... Dr. Greene 4 d 82. O clap your hands ... J.Stainer $6 \mathrm{~d}$ 80. O clap your hands ...E. H. Thorne $6 \mathrm{~d}$. 686. O clap your hands J. L. Hopkins $3 d$. 656. O come and behold

202. O come before W. H. Longhurst $4 \mathrm{~d}$. W. Martin 6d. 569. O come, let us sing M. B. Foster 12. O come near to the Cross Gounod 8d II. O day of penitence... Gounod $6 \mathrm{~d}$. 16. O give thanks ... Sir G. Elvey 3 d. 144. O give thanks $\quad . . . \quad H$. Purcell $6 \mathrm{~d}$. 17. O give thanks .... William Rea 3 d. 66. O give thanks _... S.S. Wesley 4 d. 42. O give thanks ...Sir John Goss 3 d. 520. O give thanks $\quad . . \quad$ B. Steane $3 d$. 599. O give thanks $\quad \ldots \quad$ E. V. Hall 3 d. 596. O give thanks $\ldots$ H. J. King 3 d. 35. O God, have mercy... J. B. Calkin 4d. 698. O God, my soul ... F. R. Greenish 3d 106. O God, the King of Glory H. Smart 4 d 14I. O God, Thou art my God H. Purcell 3 d. 585. Ditto B. L. Selby $4 \mathrm{~d}$. 679. O God, Thou art my God F. Tozer 4 d 34. O God, Thouart worthy A.Sullivar 4d I88. O God, Thou hast ... H. Purcell td 4I8. O God, Who hast ... A. S. Baker 2d. 430. Ditto A. W. Batson 3d 507. Ditto J. V. Roberts 2d. 47. O how amiable $\cdots$ J. Barnby $3 \mathrm{~d}$ 233. O how amiable T. M. Pattison 3d. 347. O how amiable $\ldots$ Oliver King $4 \mathrm{~d}$. 48. O how plentiful T. M. Pattison 3d. 49o. O Jerusalem, look about E. Naylor 4d. 30r. O Jesu! Victim Rev. J. B. Powell 3d. 536. O joyful Light ... B. Tours 4 d. 543. O Lamb of God ... J. Barnby 3d. 25I. O Lord God ... Ernest Ford 4d 204. O Lord God ... ... $\quad$ Dr. Clarke 3 d. 184. O Lord, look down .... J. Battishill 3 d. 702. O Lord, my God $\quad \ldots \quad$ Nares 3 d. 306. O Lord of hosts ... Kate Boundy $3 \mathrm{~d}$. 25. O Lord, our Governor H. Gadsby 3d. 395. O Lord, Thou art ... Ch. H. Lloyd 6d. 690. O Lord, Thy Word J. F. Bridge 6d. 588. O lovely peace ... Handel 4 d 3. O love the Lord Sir A. Sullivan 2d. 460. O my heart was glad A. Carnall 4 d. 556. Open to me the gates ... F. Adlam 4d. 380. O perfect love $\quad . . \quad$ J. Barnby $3 \mathrm{~d}$. 124. O praise God ... Dr. Clarke 6d. 40. O praise God T. T. Trimnell $4 \mathrm{~d}$. 429. O praise God ... G. C. Martin 2d. 355. O praise God Theodore Distin 3d. 479. O praise our God, ye people Boyce 4 d. 14. O praise the Lord ... J. Barnby 4 d. 683. O praise the Lord ... Handel 4d. 232. O praise the Lord T. M. Pattison 3d. 178. O praise the Lord ...Sir John Goss 6d. 71. O praise the Lord ... Ouseley 3d. 266. O praise the Lord ... Z Zingarelli $8 d$ 358. O praise the Lord W. G. Wood 4 d. I66. O pray for the peace E. H. Thorne 4 d. 5r. O Saving Victim(No. 2) Gounod 4 d. 486. Ditto ... Rossini 3d. 492. Ditto W.A.C. Cruickshank 3d. 508. O Saviour of the world (A.T.T.B.) J. V. Roberts 2d. 142. O sing unto the Lord H. Purcell $6 d$ 55I. O sing unto the Lord Cruickshank $4 \mathrm{~d}$. 8. O taste and see ....Sir John Goss 3d. 8*. Ditto (Welsh Words) ‥ 3d. 263. O taste and see ... A. H. Mann 3 d. 87. O that I knew where I might find him ... Sir W. S. Bennett 3d. 663. O Voice of the Beloved H. J. King 3 d. 123. O where shall wisdom Dr. Boyce 6d. 435. O worship the King... E. V. Hall 4 d.

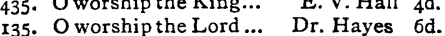
135. O worship the Lord... Dr. Hayes
158. O ye that love the Lord Sir G. Elvey ${ }_{4}$ d. 673. Now sinks the sun H. W. Parker 4d. Ig6. Ditto H. W. Wareing 3d.

LONDON : NOVELLO AND COMPANY, LiMITED. 